\title{
Frequent hypermethylation of a CTCF binding site influences Wilms tumor 1 expression in Wilms tumors
}

\author{
FERDINAND ZITZMANN ${ }^{1}$, DORIS MAYR ${ }^{2}$, MICHAEL BERGER ${ }^{1}$, MAXIMILIAN STEHR $^{1}$, \\ DIETRICH VON SCHWEINITZ ${ }^{1}$, ROLAND KAPPLER ${ }^{1}$ and JOCHEN HUBERTUS ${ }^{1}$ \\ ${ }^{1}$ Department of Pediatric Surgery, Research Laboratories and ${ }^{2}$ Institute of Pathology, \\ Ludwig-Maximilians University of Munich, D-80337 Munich, Germany
}

Received November 29, 2013; Accepted January 3, 2014

DOI: $10.3892 /$ or.2014.3019

\begin{abstract}
The Wilms tumor 1 (WT1) gene plays an essential role in early development and differentiation of the urinary tract, particularly the kidneys. Aberrant transcriptional activity of WT1 is a key finding in the genesis of Wilms tumors (WTs). However, the mechanisms responsible for this alteration remain poorly understood. In the present study, we examined the methylation pattern of a putative CCCTCbinding factor (CTCF) binding site downstream of the WT1 gene as a potential cause of WT1 misregulation in 44 native WT specimens. We found that $16 \mathrm{WT}$ cases exhibited a much higher WT1 expression compared to normal kidney tissue, and that the high mRNA expression of WT1 is strongly correlated with a high degree of DNA methylation of the CTCF binding site near the WT1 promoter. However, there was no correlation between the KTS+/KTS- splicing variants of WT1 and the methylation status of the CpGs of the CTCF binding site. Our results demonstrated an aberrant methylation pattern at a CTCF binding site downstream the WT1 gene, which is associated with an elevated WT1 transcriptional activity. Thus, methylation of the CTCF binding site may be partially responsible for the transcriptional activation of the WT1 locus and hypermethylation of this site may be an important oncogenic mechanism in the genesis of WT.
\end{abstract}

\section{Introduction}

The Wilms tumor 1 (WT1) gene has, among others, an essential role in early development and differentiation of the urinary tract, particularly the kidneys $(1,2)$. Mutations of WT1 were first described in Wilms tumors (WTs) of patients with Wilms tumor, aniridia, genitourinary anomalies, and mental

Correspondence to: Dr Jochen Hubertus, Department of Pediatric Surgery, Research Laboratories, Ludwig-Maximilians University of Munich, Lindwurmstrasse 2a, D-80337 Munich, Germany E-mail: jochen.hubertus@med.lmu.de

Key words: Wilms tumor, Wilms tumor 1, methylation, CCCTCbinding factor retardation (WAGR syndrome) (3), and the function of WT1 is believed to be that of a tumor suppressor gene (TSG). Several studies have shown a crucial role of WT1 in other types of cancer and syndromes such as acute myeloic leukemia (AML), Denys-Drash syndrome, and Frasier syndrome. While genetic alterations, such as mutations, deletions and/or truncations of WT1, are consistently detectable in those syndromes, only 20-30\% of previously described sporadic WTs are positive for WT1 anomalies (4).

Therefore, while playing a key role in early development, recent studies have shown that WT1 not only functions as a TSG, but also as an oncogene $(1,5,6)$. Two predominant splice isoforms of WT1 have been described to be responsible for these different functions; the first is characterized by omitting exon 5 and thus 17 amino acids of the WT1 protein, which are inserted between the proline-rich amino terminus and the zinc finger domains (7). The second results from alternative splicing of exon 9 that produces an insertion of the three amino acids, lysine, threonine and serine (KTS) between zinc fingers 3 and 4 . While the functional impact of exon 5 splicing remains unknown, the KTS isoforms have different affinity to the DNA $(5,8-10)$. These isoforms are expressed in a stable ratio of 2:1 (KTS+/KTS-) in healthy tissues (8).

However, (mis-)regulation of WT1 in pathological conditions is only partially understood. One well-known regulatory mechanism in WT is the methylation dependent (in-) activation of imprinted genes (11). Some imprinted genes are regulated by methylation of regulatory regions inside of $\mathrm{CpG}$-rich sites which leads to a subsequent inability of the CCCTC-binding factor (CTCF) protein to bind to its designated site (12).

CTCF is a ubiquitously expressed and highly conserved 11-zinc finger transcription factor, which is known to have diverse regulatory functions all over the human genome. Depending on the locus, CTCF can be a promoter associated transcriptional repressor or activator. Moreover, CTCF is involved in long range processes such as chromatin looping, chromatin insulation, enhancer blocking, establishing interchromosomal contacts and especially at imprinted loci, protection against ectopic de novo methylation $(13,14)$.

WT1 is expressed from both alleles in human kidney; however, it can also be imprinted in human uterus or fetal brain (15). Although the WT1 promoter seems to be unmethylated in WTs (16), previous studies suggest an epigenetic 
regulation for the WT1 antisense transcript and an alternative transcript of WT1 (AWT1), which is related to a CTCF binding site (17).

The aim of the present study was to analyze the methylation pattern at regulatory elements of the WT1 gene in a cohort of WT patients and to compare its influence on gene expression and KTS splice variants.

\section{Materials and methods}

Patients. Forty-four native WT specimens were examined from patients undergoing surgical tumor resection in our department. The median age at time of surgery was 34.5 months (range, 2 months-17 years) with a gender ratio of 1:1.5 (f:m). Thirty-seven patients (82\%) underwent neoadjuvant chemotherapy according to the International Society of Pediatric Oncology (SIOP) protocol (18). Eleven patients (24\%) were found to have bilateral WT. The control group $(n=11)$ consisted of renal tissue from the healthy part of the resected specimen after tumor nephrectomy. These controls are labeled as 'control' throughout the text. The median age of the control group was 39 months (range, 3 months-5 years) with a gender ratio of 1:2.7 (f:m). A trained pathologist performed histological classification of the samples. The study was approved by the local Ethics Committee of the Ludwig-Maximilians University, Munich. Written consent was obtained from all parents.

Search for candidate CTCF binding sites. We searched for CTCF binding sites in the surrounding of WT1 by investigating the following criteria: i) similarity to the consensus of the CTCF core sequence $(19 \mathrm{bp})$ from the JASPAR ${ }^{\circledR}$ database according to position weight matrix (PWM); ii) confirmation of the putative CTCF binding site through ChIP-sec data sets included in the Ensemble ${ }^{\circledR}$ regulatory database (human regulatory built 11 ); iii) localization in a $\mathrm{CpG}$-rich area. Following these criteria, we found one candidate site which has a PWM-score of 0.93, a confirmed CTCF binding in 24 ChIP-seq data sets in 11 different human cell lines, and is located in an area with a $\mathrm{CpG}$ ratio $>60 \%$.

Real-time reverse transcription-PCR (RT-PCR). TRI reagent ${ }^{\circledR}$ was used for isolation of total RNA from native samples. Total RNA was depleted from DNA and subsequently purified using DNase set and RNeasy Mini kit, respectively (Qiagen, Hilden, Germany). Reverse transcription of total RNA was performed using random hexamers (Roche Diagnostics, Penzberg, Germany) and SuperScript II reverse transcriptase (Invitrogen, Carlsbad, CA, USA). Intron-spanning primers were designed for the human gene WT1 using Primer Express ${ }^{\circledR}$ v2.0 (Applied Biosystems, Foster City, CA, USA) based on the sequence information contained in the Ensembl database. Primers specific for all transcripts are listed in Table I. PCR amplifications were carried out with $40 \mathrm{ng}$ of cDNA, $500 \mathrm{nM}$ forward and reverse primer and iTaq SYBR-Green Supermix (Bio-Rad Laboratories, Hercules, CA, USA) in a final volume of $20 \mu 1$. PCR reactions were run for 40 cycles consisting of $15 \mathrm{sec}$ denaturation at $95^{\circ} \mathrm{C}$, primer annealing for $15 \mathrm{sec}$ at $55^{\circ} \mathrm{C}$, and extension for $30 \mathrm{sec}$ at $72^{\circ} \mathrm{C}$ on a Mastercycler Realplex ${ }^{2}$ cycler (Eppendorf, Hamburg, Germany). All PCR reactions were
Table I. Primers used for gene expression, DNA methylation and splice variant analyses.

\begin{tabular}{|c|c|}
\hline $\begin{array}{l}\text { Gene expression } \\
\text { via RT-PCR }\end{array}$ & Sequence $\left(5^{\prime}->3^{\prime}\right)$ \\
\hline WT1 fw & CGAGAGCCAGCCCGCTA \\
\hline WT1 rv & TCGAAGGTGACCGTGCTGTA \\
\hline TBP fw & GCCCGAAACGCCGAATAT \\
\hline TBP rv & CCGTGGTTCGTGGCTCTCT \\
\hline \multicolumn{2}{|l|}{$\begin{array}{l}\text { Methylation } \\
\text { analysis via } \\
\text { pyrosequencing }\end{array}$} \\
\hline WT1 f1 & GAAAGGTTATTAGGTATTGTGTTAAGG \\
\hline WT1 r1 & ATACCACTAАATATCСТCACATATACAC \\
\hline WT1 s1 & AAAGTATTTGTTTTTTATATTGAG \\
\hline
\end{tabular}

Splice variant

analysis via

pyrosequencing

\section{WT1_KTS_F TCCGACCACCTGAAGACC \\ WT1_KTS_R ACAACTTGGCCACCGACAG WT1_KTS_Pyro ACACCAGGACTCATACAG}

fw, forward; rv, reverse.

prepared in doublets and standardized to the reference gene TATA box-binding protein (TBP). Level of expression was calculated according to the mathematical model of Pfaffl (19).

Quantification of CTCF binding site methylation using Pyrosequencing ${ }^{\circledR}$. Genomic DNA was extracted from native samples using standard procedures and converted using the EpiTect Bisulfite kit ${ }^{\circledR}$ (Qiagen) according to the manufacturer's manual.Pyrosequencing primers were designed with PyroMark Assay Design Software 2.0 and are provided in Table I. PCR was carried out using $50 \mathrm{ng}$ DNA, $500 \mathrm{nM}$ forward and reverse primer, and Maxima HotStart Taq (Fermentas, Glen Burnie, $\mathrm{MD}, \mathrm{USA}$ ) in a final volume of $15 \mu \mathrm{l}$. PCR reactions were run for 45 cycles consisting of $20 \mathrm{sec}$ denaturation at $95^{\circ} \mathrm{C}$, primer annealing for $20 \mathrm{sec}$ at $58.3^{\circ} \mathrm{C}$, and extension for $35 \mathrm{sec}$ at $72^{\circ} \mathrm{C}$ on a Mastercycler Realplex ${ }^{2}$ cycler (Eppendorf). PCR products were sequenced on a Qiagen PyroMark ${ }^{\mathrm{TM}}$ Q24 instrument using PyroMark Gold reagents and PyroMark Q24 Vacuum Prep Workstation (Qiagen) as recommended by the manufacturer. Quantitative analysis of methylation was accomplished using the Pyro Q-CpG Software (Qiagen).

Limits for hyper-respective hypomethylation were considered as two-fold the standard deviation $( \pm 2 \mathrm{SD})$.

Analysis of WT1 mRNA KTS+/KTS- splice variants by Pyrosequencing. Pyrosequencing primers were designed to flank the KTS+/KTS- splicing event of exon 9 using PyroMark Assay Design Software 2.0 (Table I). PCR results in two alternative DNA amplification products, which were subsequently 


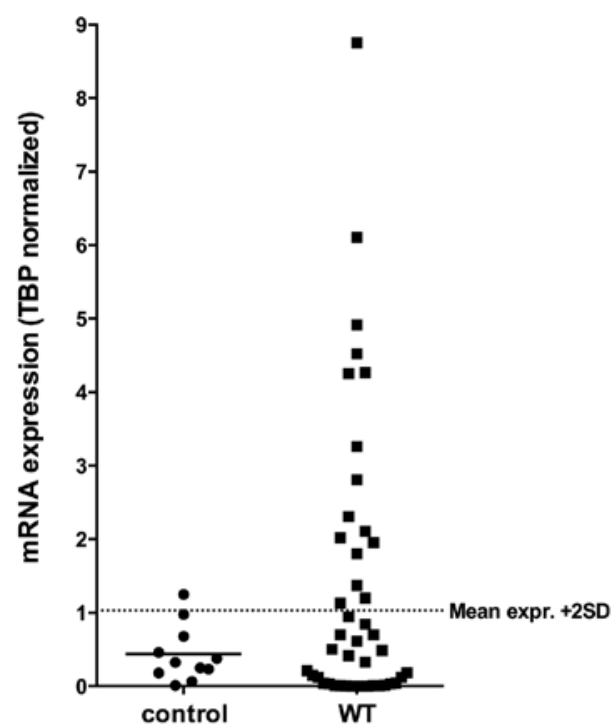

Figure 1. WT1 mRNA expression of WT and controls. No significant difference was observed for WT1 expression. However, a group of WTs showed a high transcriptional activity. Dotted line depicts 2 -fold of the standard deviation (SD) of the mean WT1 expression of controls. WT1, Wilms tumor 1.

sequenced as described above to determine their relative proportion. The KTS+/KTS- ratio was calculated according to Méreau et al (20).

Statistical analysis. Statistical analysis was performed with SPSS $^{\circledR}$ v19.0. An explorative analysis was made without corrections of p-values for multiple testing. Student's t-test was used for comparison of gene expression data and methylation status. Graphs and figures were created using Graphpad Prism version 6 .

\section{Results}

WT1 mRNA expression is upregulated in WT. First, we investigated the transcriptional activity of WT1 in a collection of 44 WTs and compared it to renal control samples. Statistical analysis revealed that there is no difference of mean WT1 expression between tumors and controls $(\mathrm{p}=0.64)$. However, $16(36.4 \%)$ WT cases showed a much higher WT1 expression the mean expression +2 SD of controls (Fig. 1).

DNA hypermethylation of the CTCF binding site in WT. To analyze the methylation status at the CTCF binding site, we designed primers that cover a $60 \mathrm{bp}$ DNA segment containing 6 CpGs (Fig. 2A). Investigating the degree of DNA methylation we revealed a mean methylation rate of $54.5 \%$ for all tumors, whereas that of controls was $40.0 \%$ (Fig. 2B). While DNA methylation level of all tested controls showed only slight variations (range from 37.0 to $43.7 \%$ ), DNA methylation of tumors varied extremely (range from 11.0 to $80.8 \%$ ).

Notably, mean methylation at the individual $\mathrm{CpGs}$ differed considerably, with a range from $65.5 \%$ at $\mathrm{CpG} 1$ to $23.5 \%$ at CpG4 in controls (Fig. 2C). However, differences in DNA methylation between tumors and controls was observed only at CpGs 2-4 (Fig. 2C), which span the core CTCF-binding site.

Considering only the three differentially methylated CpGs, we divided our samples into two groups; one contained tumors with a normal CTCF methylation, and the other contained hypermethylated tumors, using $31.95 \%$ methylation as a cut-off value (mean methylation of the CpGs 2-4 in controls $+2 \mathrm{SD}$ ). The normal methylated group contained 12 (27.3\%) samples with a mean methylation of $27.2 \%$ for $\mathrm{CpG} 2-4$. The second group contained $32(72.7 \%)$ samples, which we considered as hypermethylated. The mean methylation rate for these tumors was $59.8 \%$ (Fig. 3A).
A
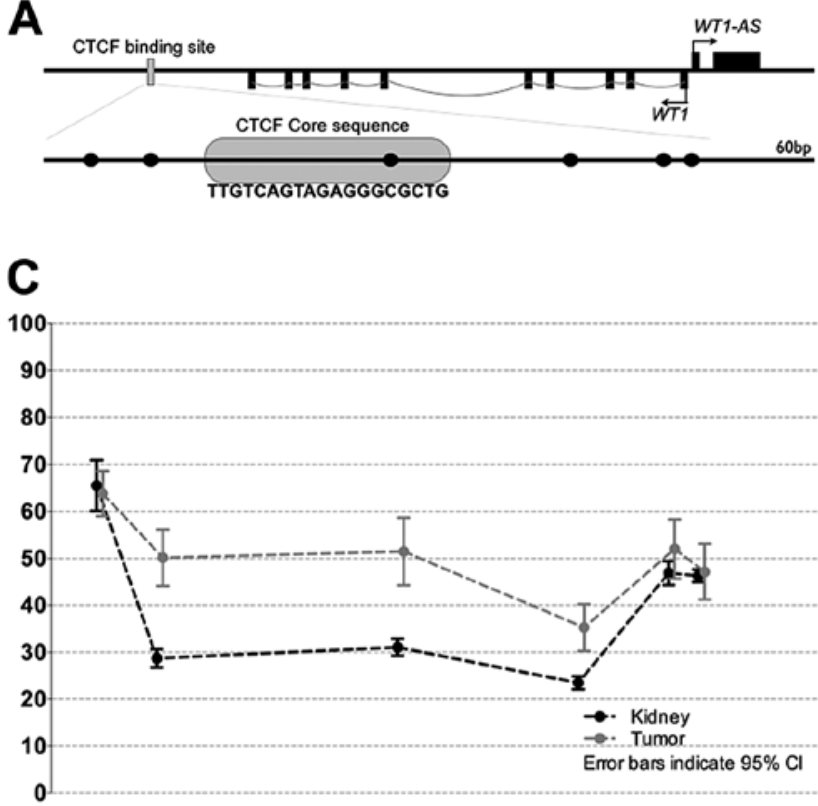

B

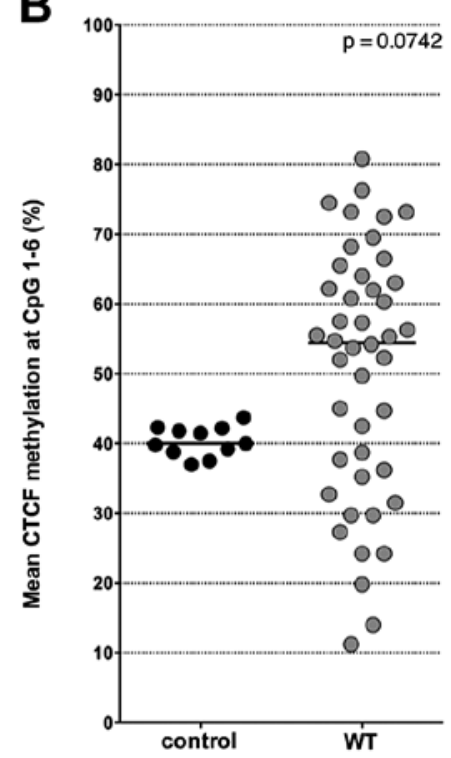

Figure 2. (A) Overview of the WT1 locus including the examined CTCF binding site located $\sim 60 \mathrm{kbp}$ downstream the WT1 gene. Detailed sequence of the CTCF binding site containing 6 CpGs is highlighted. (B) Mean methylation of CpGs 1-6 in normal kidney and tumor cases with median depicted as a black line. (C) Mean methylation at each single CpG near the CTCF binding site plotted vs. controls. Differences in methylation were observed especially at CpG 2-4 with higher methylation levels in WT. WT1, Wilms tumor 1; CTCF, CCCTC-binding factor. 

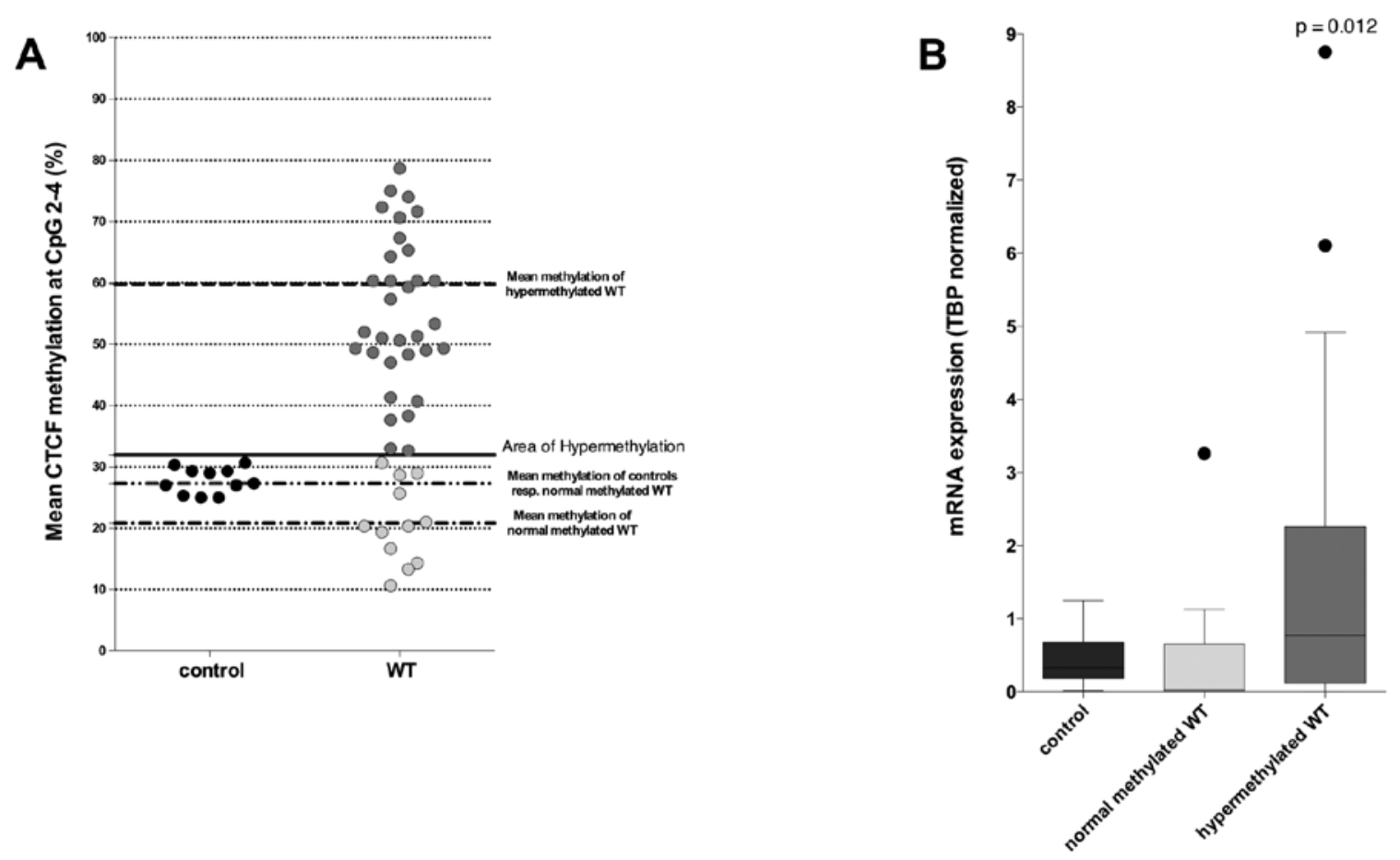

Figure 3. (A) Distribution of mean DNA methylation at the CpGs 2-4. Note the wide range of tumor methylation in comparison to the stable methylation pattern in controls. Tumors were classified either as normal or hypermethylated. The cut-off level was defined as + 2SD of the mean methylation in controls. (B) WT1 mRNA expression of controls and WTs with a hypermethylation of the CpGs 2-4. The hypermethylated WTs have a significantly higher level of mRNA expression compared to control. mRNA expression of normal methylated WTs are equal to control. WT1, Wilms tumor 1.

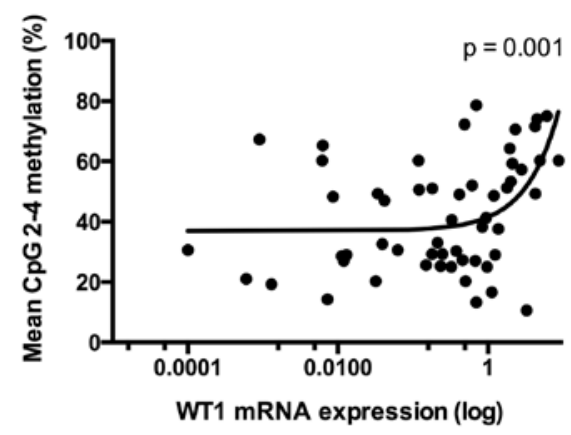

Figure 4. Correlation of mean $\mathrm{CpG} 2-4$ methylation and transcriptional activity of WT1. Results are presented in logarithmic scale. WT1, Wilms tumor 1 .

Hypermethylated tumors showed significant overexpression of WT1 compared to normal methylated tumors $(\mathrm{p}=0.012)($ Fig. 3B). There was a relevant correlation $(\mathrm{r}=0.435)$ between mean methylation of the CpGs 2-4 and the mRNA expression of WT1 (p=0.001) (Fig. 4).

KTS+/KTS- splicing variants are not differentially expressed in WT. Ratio for KTS+/KTS- transcript was found to be stable in healthy tissues at a value of $2: 1(7,21)$. We determined the median KTS+/KTS- ratio to be 1.86:1 for controls and 1.97:1 for WTs, respectively (Fig. 5). Apart from four outliers with a ratio $>2.5: 1$ and one outlier with a ratio of 1:1, ratios presented to be quite constant in both tumors and controls. Consequently, no statistically relevant deviation between transcript ratios of WT and controls were found (Fig 5). Similarly, there was no

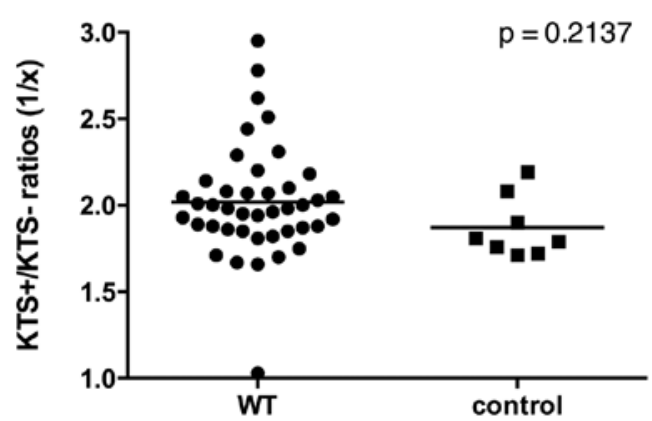

Figure 5. Ratios of KTS+ to KTS- transcripts in WTs and controls. No differences were detectable between WTs and controls. Ratios are presented as 1/x. WT, Wilms tumor.

statistical correlation between the KTS+/KTS- ratios and the methylation status of the CpGs 2-4 at the CTCF binding sites $(\mathrm{p}=0.914)$, but a trend toward a correlation of KTS+/KTSratios and WT1 mRNA expression ( $\mathrm{p}=0.061$, data not shown).

\section{Discussion}

The outstanding role of WT1 in urinary tract development is well known. Its function as a TSG was assumed due to the findings that mutations of WT1 led to both anomalies in the urinary tract and malignancies in different tissues, particularly in kidneys. However, mutations of WT1 are detectable in approximately $30 \%$ of sporadic WTs, only. Moreover, it is known that WT1 mRNA is overexpressed in a large number of WTs. In our study, 16 (36.4\%) tumors showed WT1 overex- 
pression, further casting the generally perceived doubt on an exclusive function of WT1 as a TSG. In fact, previous studies indicated that specific WT1 splice variants can act as an oncogene. The KTS+/KTS- splicing variants in particular seem to be relevant in this manner (21).

However, mechanisms other than mutation of TSG are known to be involved in tumorigenesis, particularly in childhood tumors. We previously described that methylation of imprinted genes is disturbed in a large number of WTs (22). While WT1 is not exclusively expressed from a single allele in human kidney, it can be imprinted in selected human tissues. Jinno et al reported a biallelic or exclusively maternal expression in fetal brain and uterus (15). Mitsuya et al confirmed a paternal imprinting in human fibroblasts and lymphocytes in some cases (23). Malik et al and later Hancock et al reported an epigenetic regulation of a WT1 antisense transcript $(17,24)$. An alternative transcript of WT1 (AWT1) has also been described (24), which may undergo loss of imprinting during WT tumorigenesis (25).

Considering these data, we investigated an impact of DNA methylation on WT1 mRNA expression. As we have demonstrated the influence of CTCF binding site methylation on both NNAT and IGF2/H19 loci (12), we searched for such a binding site in the surrounding of WT1. The detected binding site is located downstream the WT1 gene and inside a CpG island. Correlating the methylation with the WT1 expression, we displayed a distinct overexpression for tumors with hypermethylation at this site, whereas tumors with normal methylation pattern had an mRNA expression equal to the control. Renda et al confirmed that the affinity of the CTCF factor may be inhibited by methylation of specific $\mathrm{CpGs}$ in the CTCF core sequence in chicken $\beta$-globulin (26). In addition, $\mathrm{Xu}$ et al described that even flanking $\mathrm{CpGs}$ in the surrounding of a CTCF core sequence affects the accessibility of CTCF at the breast cancer 1 (BRCA1) promoter (27). According to these findings, we hypothesized that the hypermethylation of the CTCF core binding site has an effect on the transcriptional activity due to a lower affinity of CTCF to its binding site. In line with this, we observed that only the CpGs 2-4 that span the CTCF binding site had higher levels of methylation in tumors compared to controls (Fig. 2). These findings are consistent with a theory proposed by Phillips and Corces that describes a retaining of the $\mathrm{CTCF}$ factor during the whole cell cycle to protect its binding sites against DNA methylation, particularly those involved in DNA looping (13).

Although aberrant WT1 expression is a common phenomenon in WTs, another mechanism may also be involved in tumorigenesis, which is the different functionality of WT1 transcripts due to the KTS+/KTS- splice variants. Thus, we investigated whether CTCF is not only involved in the regulation of the expression, but also in the maintenance of the $\mathrm{KTS}+/ \mathrm{KTS}$ - ratio. In our analysis, there was no statistical correlation between the methylation of the CTCF binding site and the KTS+/KTS- ratio $(\mathrm{p}=0.941)$, but a trend toward a correlation between WT1 mRNA expression and the KTS+/ $\mathrm{KTS}$ - ratio $(\mathrm{p}=0.061)$. This stands for an independent regulation of the KTS+/KTS- ratio from the CTCF binding site methylation. Even if there is a true correlation between the KTS+/KTS- ratio and the WT1 mRNA expression, this cannot be extracted from our data at this time and opens the field for further investigations.
In conclusion, we have detected an aberrant methylation pattern at a CTCF binding site downstream the WT1 gene, which is associated with an elevated WT1 transcriptional activity. However, regulation of the KTS+/KTS- transcripts is independent of the CTCF binding site methylation. Thus, methylation of the CTCF binding site may be partially responsible for the transcriptional activation of the WT1 locus and hypermethylation of this site may be one important oncogenic mechanism in WT.

\section{References}

1. Hohenstein P and Hastie ND: The many facets of the Wilms' tumour gene, WT1. Hum Mol Genet 15: R196-R201, 2006.

2. Rivera MN and Haber DA: Wilms' tumour: connecting tumorigenesis and organ development in the kidney. Nat Rev Cancer 5: 699-712, 2005.

3. Pelletier J, Bruening W, Li FP, et al: WT1 mutations contribute to abnormal genital system development and hereditary Wilms' tumour. Nature 353: 431-434, 1991.

4. Brown KW, Wilmore HP, Watson JE, et al: Low frequency of mutations in the WT1 coding region in Wilms' tumor. Genes Chromosomes Cancer 8: 74-79, 1993.

5. Wells J, Rivera MN, Kim WJ, Starbuck K and Haber DA: The predominant WT1 isoform (+KTS) encodes a DNA-binding protein targeting the planar cell polarity gene Scribble in renal podocytes. Mol Cancer Res 8: 975-985, 2010.

6. Yang L, Han Y, Suarez Saiz F, Saurez Saiz F and Minden MD: A tumor suppressor and oncogene: the WT1 story. Leukemia 21: 868-876, 2007.

7. Haber DA, Sohn RL, Buckler AJ and Housman DE: Alternative splicing and genomic structure of the Wilms tumor gene WT1. Proc Natl Acad Sci USA 88: 9618-9622, 1991.

8. Klamt B, Koziell A, Poulat F, et al: Frasier syndrome is caused by defective alternative splicing of $W T 1$ leading to an altered ratio of WT1 +/-KTS splice isoforms. Hum Mol Genet 7: 709-714, 1998.

9. Bor YC, Swartz J, Morrison A, Rekosh D, Ladomery M and Hammarskjöld ML: The Wilms' tumor 1 (WT1) gene (+KTS isoform) functions with a CTE to enhance translation from an unspliced RNA with a retained intron. Genes Dev 20: 1597-1608, 2006.

10. Hammes A, Guo JK, Lutsch G, et al: Two splice variants of the Wilms' tumor 1 gene have distinct functions during sex determination and nephron formation. Cell 106: 319-329, 2001.

11. Lewis A and Reik W: How imprinting centres work. Cytogenet Genome Res 113: 81-89, 2006.

12. Hubertus J, Zitzmann F, Trippel F, et al: Selective methylation of CpGs at regulatory binding sites controls NNAT expression in Wilms tumors. PLoS One 8: e67605, 2013.

13. Phillips JE and Corces VG: CTCF: master weaver of the genome. Cell 137: 1194-1211, 2009.

14. Fiorentino FP and Giordano A: The tumor suppressor role of CTCF. J Cell Physiol 227: 479-492, 2012.

15. Jinno Y, Yun K, Nishiwaki K, et al: Mosaic and polymorphic imprinting of the WT1 gene in humans. Nat Genet 6: 305-309, 1994.

16. Mares J, Kríz V, Weinhäusel A, et al: Methylation changes in promoter and enhancer regions of the WT1 gene in Wilms tumours. Cancer Lett 166: 165-171, 2001.

17. Hancock AL, Brown KW, Moorwood K, et al: A CTCF-binding silencer regulates the imprinted genes $A W T 1$ and $W T 1-A S$ and exhibits sequential epigenetic defects during Wilms' tumourigenesis. Hum Mol Genet 16: 343-354, 2007.

18. de Kraker J, Graf N, van Tinteren H, et al: Reduction of postoperative chemotherapy in children with stage I intermediate-risk and anaplastic Wilms' tumour (SIOP 93-01 trial): a randomised controlled trial. Lancet 364: 1229-1235, 2004.

19. Pfaffl MW: A new mathematical model for relative quantification in real-time RT-PCR. Nucleic Acids Res 29: e45, 2001.

20. Méreau A, Anquetil V, Cibois M, et al: Analysis of splicing patterns by pyrosequencing. Nucleic Acids Res 37: e126, 2009.

21. Burwell EA, McCarty GP, Simpson LA, Thompson KA and Loeb DM: Isoforms of Wilms' tumor suppressor gene (WT1) have distinct effects on mammary epithelial cells. Oncogene 26: 3423-3430, 2007. 
22. Hubertus J, Lacher M, Rottenkolber M, Müller-Höcker J, Berger M, et al: Altered expression of imprinted genes in Wilms tumors. Oncol Rep 25: 817-823, 2011.

23. Mitsuya K, Sui H, Meguro M, Kugoh H, Jinno Y, et al: Paternal expression of WT1 in human fibroblasts and lymphocytes. Hum Mol Genet 6: 2243-2246, 1997.

24. Malik K and Brown KW: Epigenetic gene deregulation in cancer. Br J Cancer 83: 1583-1588, 2000.

25. Brown KW, Power F, Moore B, Charles AK and Malik KT: Frequency and timing of loss of imprinting at $11 \mathrm{p} 13$ and $11 \mathrm{p} 15$ in Wilms' tumor development. Mol Cancer Res 6: 1114-1123, 2008.
26. Renda M, Baglivo I, Burgess-Beusse B, Esposito S, Fattorusso R, et al: Critical DNA binding interactions of the insulator protein CTCF: a small number of zinc fingers mediate strong binding, and a single finger-DNA interaction controls binding at imprinted loci. J Biol Chem 282: 33336-33345, 2007.

27. Xu X, Gammon MD, Zhang Y, Cho YH, Wetmur JG, et al: Gene promoter methylation is associated with increased mortality among women with breast cancer. Breast Cancer Res Treat 121: 685-692, 2010. 